\title{
Investigation of Allergy Management in Primary Care: Child vs. Adult Prescriptions
}

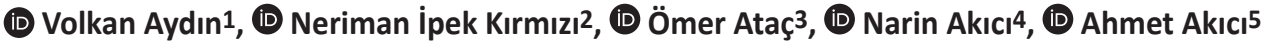 \\ 1istanbul Medipol University International School of Medicine, Department of Medical Pharmacology, Istanbul, Turkey \\ 2 istanbul Medipol University School of Medicine, Department of Medical Pharmacology, Istanbul, Turkey \\ 3 Istanbul Medipol University School of Medicine, Department of Public Health, Istanbul, Turkey \\ ${ }^{4}$ Haydarpaşa Numune Training and Research Hospital, Clinic of Pediatrics, Istanbul, Turkey \\ ${ }^{5}$ Marmara University School of Medicine, Department of Medical Pharmacology, Istanbul, Turkey
}

\section{Abstract}

Objective: Allergic diseases are conditions that are frequently encountered in primary care, and different drug groups can be used in their treatment. This study aimed to compare the use of drugs in allergy in children and adults applied to primary care.

Methods: We analyzed prescriptions written by those who were selected by systematic sampling $(n=1431)$ among family physicians serving in İstanbul between January 1 and December 31, 2016. Among these, single-diagnosis prescriptions containing "T78.4-allergy, unspecified" were included in the study, and the prescriptions were divided into those written to children ( $<18$ years old) and adults ( $\geq 18$ years old). The demographic characteristics of the patients and drug details in the prescriptions were compared according to the groups.

Results: A total of 37,042 prescriptions with a single diagnosis of allergy were identified, and 55.9\% of which were for adults. Allergy diagnosis was higher in men (52.4\%) among children and in females (67.7\%) among adults. Antihistamines (85.3\% and 83.4\%, p <0.001), systemic steroids $(5.4 \%$ and $1.6 \%, p<0.001)$, and inhalants $(1.8 \%$ and $1.3 \% ; p<0.001)$ were more likely prescribed to adults, whereas topical drugs were prescribed more in children $(51.7 \%$ and $42.7 \%, p<0.01)$. Monotherapy was more preferred in children (45.8\%) than in adults (41.6\%, $\mathrm{p}<0.0001)$. Although antihistamine monotherapy was similar in these groups, topical drug monotherapy was used more in children (10.3\%) than in adults (5.6\%). Prescriptions with first-generation antihistamines were higher in adults (6.8\%) than in children (5.4\%; $\mathrm{p}<0.001)$. Desloratadine was the most commonly encountered drug in the prescriptions of both pediatric and adult patients (21.2\% and $10.3 \%$, respectively).

Conclusion: The study revealed that antihistamines, mostly second-generation agents, are frequently preferred. Apart from the higher prescription of systemic corticosteroids for adults and topical drugs for children, it is understood that the pharmacological management of allergic conditions in primary care shows overall similarities in both age groups.

Keywords: Allergy, family physicians, pediatrics, adults, antihistamines, topical drugs

\section{INTRODUCTION}

Allergic diseases, which are a frequent reason for morbidity worldwide, pose a significant burden on healthcare systems. It was reported that nearly $30 \%$ of the population has been affected by allergy and allergy-related disorders and this might increase up to $80 \%$ in terms of families (1). It is common for patients to seek medical advice from primary care for their allergic conditions. Although primary care physicians are expected to manage allergic diseases appropriately, several studies in the literature showed an inadequate knowledge level with some degree of incompetency in rationally implementing the pharmacotherapy based on relevant guidelines (2-4).

Compared with adults, children require a more delicate approach in drug use and represent a special patient population whose 
dynamic physiology involves different pharmacokinetic and pharmacodynamic processes $(5,6)$. Therefore, drug utilization patterns may vary for adult and pediatric populations even in the same indication. Apart from the incidence, disease severity, and prognosis, therapeutic responses may also differ by these age groups $(6,7)$. Moreover, it is well recognized that allergic diseases are more common during childhood $(8,9)$.

About $8 \%$ of primary care visits consisted of allergic conditions (10). The disease course, outcome, and pharmacotherapy practice may be different in child and adult age groups $(5,11,12)$. Although encountered by primary care physicians often, no comprehensive study has been reflected in the literature about prescribing practices in allergy for different age groups in Turkey. In this study, we aimed to compare drug use in children versus adults who had allergy diagnoses in primary care.

\section{METHODS}

We performed a cross-sectional study where we examined prescriptions of primary care physicians between January 1 and December 31, 2016, in Istanbul. The data were collected after being approved by the non-interventional Ethics Committee of İstanbul Medipol University (approval no: 13.11.2017-521).

Of the 4.293 primary care physicians working in İstanbul, 1.431 were selected using 3:1 systematic sampling. The prescriptions with the solo diagnosis of "T78.4-allergy-unspecified" were examined retrospectively. The prescriptions with multiple diagnoses that contained allergy were not included to ensure that the specified drugs were only prescribed for allergy and, hence, to assess the appropriateness of allergy pharmacotherapy. These prescriptions were further stratified into two groups by their age groups as children ( $<18$ years old) and adults ( 218 years old).

Prescriptions generated for children and adults with allergy diagnoses were compared in terms of the mean age, gender, and monthly distribution. The number of drug items and boxes per encounter was also compared between these two age groups. The percentages of antihistamine agents, topical drugs, systemic corticosteroids, inhalants, and systemic anti-infectives in these prescriptions were identified and compared. In addition, monthly prescribing of antihistamines, systemic corticosteroids, and topical preparations were compared between children and adults. The most prescribed single agents of these drug classes were determined for each age group. The most commonly prescribed 10 drugs were identified in the child and adult groups. The percentage of patients who required systemic antihistamine plus corticosteroid combination was compared between the study groups. Furthermore, the percentage of prescriptions that contained first- and second-generation antihistamine drugs was also compared.

\section{Statistical Analysis}

We used Microsoft Office Excel 2016 and SPSS 24.0 for data analysis. Descriptive data were presented as the number and percentages or the mean and standard deviations, where appropriate. We compared the study groups via t-test and Mann-Whitney $\mathrm{U}$ test for normally and non-normally distributed continuous variables, respectively, and chi-square test for categorical variables. An overall 5\% type-I error level was used to infer statistical significance.

\section{RESULTS}

We identified 37,042 prescriptions with a single "T78.4-allergy" diagnosis. The prescriptions generated constituted $44.1 \%$ $(n=16,342)$ for children and $55.9 \%(n=20,700)$ for adults. The mean ages of children and adults were $6.7 \pm 4.5$ and $47.3 \pm 17.8$ years, respectively. The percentage of male gender in children (52.4\%) was significantly higher than in adults (32.3\%, $p<0.001)$. We detected a total of 74,380 drugs in these prescriptions, where the mean number of drug items and boxes per prescriptions was significantly lower in children $(1.8 \pm 1.0$ and $2.1 \pm 1.6)$ than in adults $(2.2 \pm 1.5$ and $3.4 \pm 4.1 ; p<0.001$ for each). The prescriptions with allergy diagnoses were most commonly generated in January for both children (12.0\%) and adults (17.8\%), and the lowest percentages were detected in December in both groups (6.0\% and 5.0\%, respectively).

Antihistamines ( $85.3 \%$ and $83.4 \%, p<0.001$ ), systemic steroids (5.4\% and $1.6 \%, p<0.001)$, and inhalants $(1.8 \%$ and $1.3 \%$, $\mathrm{p}<0.001$ ) were more likely prescribed to adults, whereas topical drugs were prescribed more in children $(51.7 \%$ and $42.7 \%$, $\mathrm{p}<0.01$ ). Allergic conditions were managed with monotherapy in $43.5 \%$ of all prescriptions, which was significantly higher in children (45.8\%) than in adults (41.6\%, $\mathrm{p}<0.0001)$. About threequarters (75.9\%) of the monotherapy included antihistamines (33.0\% of all prescriptions), followed by topical agents (17.6\% of monotherapy and $7.6 \%$ of total). Although antihistamine monotherapy was similar between children (33.0\%) and adults $(32.9 \%, p=0.81)$, topical drug monotherapy was more preferred in children $(10.3 \%)$ than in adults $(5.6 \% ; p<0.0001)$. The prescriptions with first-generation antihistamines were higher in adults (6.8\%) than in children (5.4\%; $p<0.001$; Figure 1). The need for systemic antihistamine plus corticosteroids co-prescription was significantly higher in adults (3.4\%) than in children $(1.1 \%, \mathrm{p}<0.001)$. The percentages of prescriptions 
with systemic antibiotics were similar in children (6.4\%) and adults $(6.7 \%, p>0.05)$.

Seasonal distribution of the drug utilization for allergic conditions showed that children were significantly more likely to be prescribed antihistamines between June and September and topical agents throughout the year except March compared with the adult population (Figure 2).

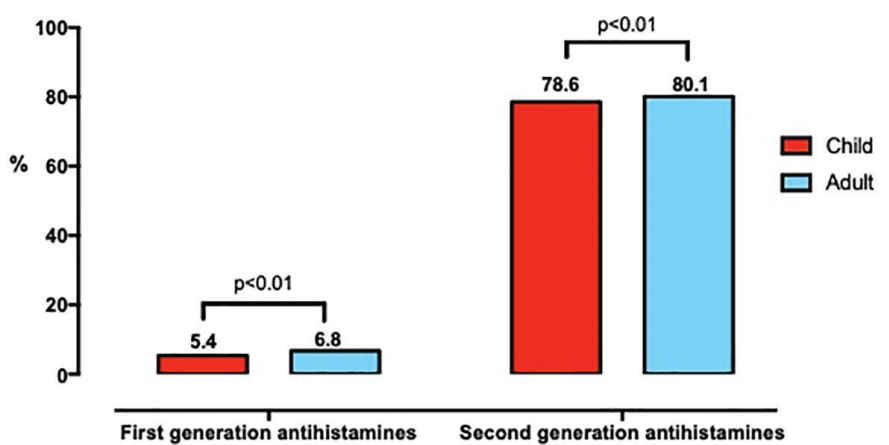

Figure 1. Comparison of prescriptions with first- and second-generation antihistamines between children and adults

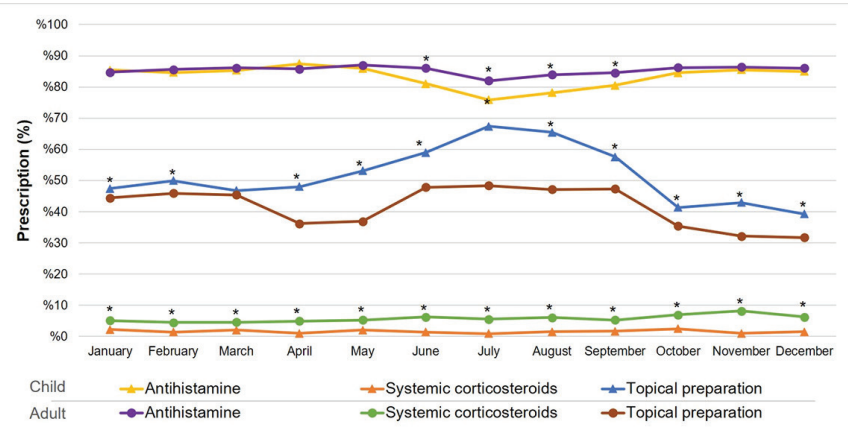

Figure 2. Monthly distribution and comparison of prescriptions with antihistamines, topical preparations, or systemic corticosteroids between children and adults. ${ }^{*} p<0.05$
The most commonly prescribed antihistamine was desloratadine in both children (45.3\%) and adults (25.1\%). "lidocaine \pm combinations" was the most commonly prescribed topical agent in these age groups (39.6\% and 21.0\%, respectively). Furthermore, methylprednisolone was the most commonly preferred systemic corticosteroid in children (53.1\%) and dexamethasone in adults (45.3\%). The most commonly encountered systemic anti-infective in allergy prescriptions were amoxicillin + enzyme inhibitor in both groups (41.9\% and 31.4\%; Table 1). Desloratadine was the most commonly encountered drug in the prescriptions of both pediatric and adult patients $(21.2 \%$ and $10.3 \%$, respectively), followed by cetirizine $(16.2 \%$ and $7.7 \%$, respectively). The third top-used drug was topical lidocaine \pm combinations in children (12.4\%) compared with rupatadine in adults (7.7\%; Figure 3).

\section{DISCUSSION}

In this study, we compared pharmacotherapy practices of primary care physicians for unspecified allergy indications in children versus adults. We observed that allergic diseases were rather managed by combination therapies mostly with secondgeneration antihistamines with higher prescription rates of
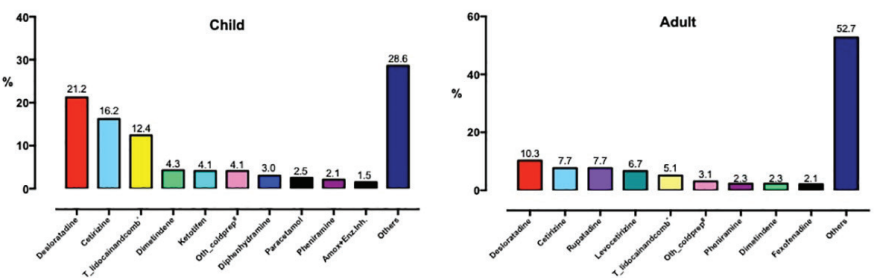

Figure 3. Distribution of all prescribed drugs for children and adults in allergy indication. *Topical lidocaine and combinations, \#other common cold preparations

\begin{tabular}{|l|l|l|l|}
\hline \multicolumn{3}{|l|}{ Table 1. Distribution of the most frequently prescribed drugs for child and adult groups } \\
\hline & Ranking & Child (\%) & Adult (\%) \\
\hline \multirow{4}{*}{ Antihistamines } & 1 & Desloratadine (45.3) & Desloratadine (25.1) \\
\cline { 2 - 4 } & 2 & Cetirizine (34.7) & Cetirizine (18.8) \\
\cline { 2 - 4 } Topical medication & 3 & Ketotifen (8.7) & Rupatadine (18.7) \\
\hline \multirow{4}{*}{ Systemic corticosteroids } & 1 & Lidocaine and combinations (39.6) & Lidocaine and combinations (21.0) \\
\cline { 2 - 4 } & 2 & Dimetindene (13.6) & Dimetindene (9.3) \\
\cline { 2 - 4 } & 3 & Diphenhydramine (9.4) & Corticosteroid + imidazole/triazole (4.1) \\
\hline \multirow{4}{*}{ Systemic anti-infectives } & 2 & Methylprednisolone (53.1) & Dexamethasone (45.3) \\
\cline { 2 - 4 } & 3 & Dexamethasone (41.3) & Methylprednisolone (36.9) \\
\cline { 2 - 4 } & 2 & Prednisolone (1.8) & Amoxicillin + enzyme inhibitor (31.4) \\
\cline { 2 - 4 } & 3 & Clarithromycin (24.4) & Clarithromycin (14.8) \\
\hline
\end{tabular}


topical agents in children and of systemic corticosteroids in adults.

With a wide spectrum from dermatitis to asthma or allergic rhinitis to anaphylaxis, allergic conditions have been reported to mostly affect the pediatric population $(8,9)$. Contrarily, our study showed a slight predominance of adults with a prescription containing an allergy diagnosis. This might be attributed to the higher utilization of primary care services by adult patients (13). Another factor might be that we only included diagnoses with unspecified allergies since children tend to manifest with symptoms of specific allergic diagnoses like asthma or rhinitis (14). Studies on the relationship between atopy and gender reported higher prevalence in men until adolescence, after which female predominance ensues under the impact of immunosuppressive testosterone and proinflammatory female sex steroids (15). In our study, the boys:girls ratio was 1.1:1 in children compared with the female:male ratio of 2:1 in adults. A primary care study performed at the national level in our country in the same year showed healthcare utilization in favor of boys by 1.1-fold in children and of women by 1.5-fold in adults (13). In this context, it can be suggested that our results may support the female predominance in allergic conditions in the adult population.

One of the mainstay pharmacological groups in managing allergic diseases is oral antihistamines $(16,17)$. The preference of antihistamines as first-line drugs for allergy indications was reported by an international survey study of primary care (2). Although slightly more pronounced in adults, both groups in our study showed frequent prescriptions of antihistamines. Although prescribing of antihistamines exhibited a similar pattern throughout the year in both groups (featuring peaks at spring months), it appears remarkable that the difference was significant during summer months. In addition, both groups mostly revealed prescriptions of second-generation antihistamines (about 80\%)-desloratadine and cetirizine-which might be regarded as non-sedating agents. Accordingly, preference of new generation antihistamines over first-generation drugs could be accepted as an overall rational prescribing practice as the latter is less safe (18). In addition, these first-generation drugs were prescribed less in children (5.4\%) than in adults (6.8\%). The fact that markedly sedative antihistamines may impair cognitive functions thereby resulting in diminished school performance (17) might also partially support the rationality of the physicians in our study in selecting new generation agents with a better safety profile. On the other hand, first-generation antihistamines also convey several risks in adults, for example, negative impacts on vehicle-device use (19). Our findings suggest that primary care physicians may tend to have a more cautious approach in children versus in adults, considering the risks relevant to antihistamines.
Corticosteroids have been used for the management of inflammatory and allergic diseases for more than 50 years. Nevertheless, their systemic use in allergic conditions remains controversial because of their adverse effects and compelling tolerability profiles $(20,21)$. Primary care physicians were reported to often prescribe systemic corticosteroids of short duration in several indications (22). Our study showed that prescriptions containing systemic corticosteroids for pediatric allergic diseases constituted near one-fifth of that in adults, although we did not have the duration of use. Unlike in adults, even the short use of corticosteroids was associated with hypothalamic-hypopituitary-adrenal axis suppression in up to $81 \%$ of children $(23,24)$. Although our study revealed a comparably lower rate of corticosteroid use (1.6\%) in children, the fact that nearly half of these agents were potent agents (i.e., dexamethasone) may suggest that such axis suppression should be considered more cautiously by physicians. This warrants further prospective research encompassing the dose and duration of corticosteroids.

Urticaria and eczema are common manifestations of allergic diseases (25). Therefore, it would be no surprise to encounter topical drugs in the symptomatic management of allergy in clinical practice. In fact, we observed a topical form in near two prescriptions, with the most frequent being topical lidocaine or its combinations in both children and adults. It is noticeable that, however, more children than adults were prescribed topical agents almost throughout the year. It might be partly attributed to the fact that challenging management of systemic agents in children due to different pharmacokinetics and pharmacodynamics might have directed physicians to prefer topical agents (5). In addition, the highest prescribing of topical agents in the summer months in both age groups may indicate a higher prevalence of insect-bite-induced allergic conditions (26).

The irrational use of antibiotics is a well-known prioritized issue of the health authority for about 10 years. Although some progress has been made owing to ongoing well-structured efforts, irrational antibiotic prescribing was often reported in terms of inappropriate indications or doses (27-29). Allergic diseases do not require the use of systemic anti-infectives unless an underlying or secondary condition entails. One of the reasons we only included the prescriptions with a solo diagnosis of allergy was to omit the rationale of prescribing antibiotics for probable secondary indications. However, it is remarkable that $7 \%$ of prescriptions with merely allergy diagnoses contained systemic anti-infective drugs both in children and adults. Furthermore, most of these agents were the amoxicillin + enzyme inhibitor, a broad-spectrum antibiotic. This substantial rate of inappropriate 
prescribing practice might provide additional critical insight for the action plans on rational antibiotic use.

The findings of our study need to be interpreted in light of its limitations. The study sample only included the prescriptions with the diagnosis of "T78.4-allergy, unspecified". Therefore, we were unable to distinguish details of drug utilization in specific allergic reactions such as urticaria, angioedema, drug/ food allergy, and insect bite, which might be listed under this unspecified indication yet managed differently. In addition, the diagnoses established by primary care physicians are regarded as definite without any further verification. Several drugs for allergic conditions, for example, some inhalants for bronchial asthma, were initiated by secondary or tertiary care physicians and managed in primary care as part of the repeated prescription practice. Such conditions might not have reflected the actual practice of primary care of allergy. Besides, we did not evaluate the detailed use of certain drug classes in specified age groups, that is, first-generation antihistamines above 65 years in adults or in school-age groups among children, which may reflect a potential of inappropriate use in clinical practice. Finally, the findings on the seasonal distribution of drug use should be carefully approached as it only represented prescriptions in 2016.

\section{CONCLUSION}

It was the first time to reveal the similarities and differences of the pharmacological management of allergy in primary care between children and adults. Although these age groups differ in terms of gender predominance, the pharmacotherapy of unspecified allergic conditions appears to be similar for children and adults, albeit with several nuances. Allergy in primary care was mostly managed by second-generation antihistamines, where one in every two patients was prescribed additional topical agents. Remarkably, the age groups featured higher prescription rates of systemic corticosteroids for adults and topical drugs for children. We believe that the findings of our study provided insights and made contributions to studies on the rational management of allergic conditions in different populations and the functionality of treatment algorithms from a primary care perspective.

\section{Ethics}

Ethics Committee Approval: The data were collected after being approved by the non-interventional Ethics Committee of İstanbul Medipol University (approval no: 13.11.2017-521).
Informed Consent: Not applicable for this study.

Peer-review: Externally peer-reviewed.

\section{Authorship Contributions}

Concept: V.A., N.I.K., Ö.A., N.A., A.A., Design: V.A., N.I.K., A.A., Data Collection or Processing: V.A., N.A., Analysis or Interpretation: V.A., N.I.K., Literature Search: V.A., N.I.K., N.A., Writing: V.A., N.İ.K., Ö.A., N.A., A.A.

Conflict of Interest: No conflict of interest was declared by the authors.

Financial Disclosure: The authors declared that this study received no financial support.

\section{REFERENCES}

1. Sánchez-Borges M, Martin BL, Muraro AM, Wood RA, Agache IO, Ansotegui IJ, et al. The importance of allergic disease in public health: an iCAALL statement. World Allergy Organ J 2018;11:8.

2. Agache I, Ryan D, Rodriguez MR, Yusuf O, Angier E, Jutel M. Allergy management in primary care across European countries -- actual status. Allergy 2013;68:836-43.

3. Uygunsoy M, Mevsim V. Diagnostic Tests in The Management of Allergic Diseases in Primary Care. Turkish J Fam Med Prim Care 2020;14:141-6.

4. Baldacci S, Maio S, Simoni M, Cerrai S, Sarno G, Silvi P, et al. The ARGA study with general practitioners: impact of medical education on asthma/rhinitis management. Respir Med 2012;106:777-85.

5. Kearns GL, Abdel-Rahman SM, Alander SW, Blowey DL, Leeder JS, Kauffman RE. Developmental pharmacology--drug disposition, action, and therapy in infants and children. N Engl J Med 2003;349:1157-67.

6. Kırmızı Ni, Aydın V, Akıcı N, Akıcı A. Çocuklarda Endikasyon Dışı İlaç Kullanımı. J Lit Pharm Sci 2020;9:149-57.

7. World Health Organization. Promoting Safety of Medicines for Children, 2007. ISBN: 9789241563437.

8. Gupta R, Sheikh A, Strachan DP, Anderson HR. Burden of allergic disease in the UK: secondary analyses of national databases. Clin Exp Allergy 2004;34:520-6.

9. Anandan C, Gupta R, Simpson CR, Fischbacher C, Sheikh A Epidemiology and disease burden from allergic disease in Scotland: analyses of national databases. J R Soc Med 2009;102:431-42.

10. Diwakar L, Cummins C, Lilford R, Roberts T. Systematic review of pathways for the delivery of allergy services. BMJ Open 2017;7:e012647

11. Wallace DV, Dykewicz MS, Bernstein DI, Blessing-Moore J, Cox L, Khan DA, et al. The diagnosis and management of rhinitis: an updated practice parameter. J Allergy Clin Immunol 2008;122(2 Suppl):S1-84.

12. Jutel M, Papadopoulos NG, Gronlund H, Hoffman HJ, Bohle B, Hellings $P$, et al. Recommendations for the allergy management in the primary care. Allergy 2014;69:708-18.

13. Bayram D, Vızdıklar C, Aydın V, İșli F, Akıcı A. Birinci basamakta reçeteleme trendi ve sık karșılașılan tanılara ait reçetelerin incelenmesi: Türkiye verisi. Cukurova Med J 2020;45:695-708. 
14. Thomsen SF. Epidemiology and natural history of atopic diseases. Eur Clin Respir J 2015;2.

15. Osman M. Therapeutic implications of sex differences in asthma and atopy. Arch Dis Child 2003;88:587-90.

16. Kuna P, Jurkiewicz D, Czarnecka-Operacz MM, Pawliczak R, Woroń $\mathrm{J}$, Moniuszko $\mathrm{M}$, et al. The role and choice criteria of antihistamines in allergy management - expert opinion. Postepy Dermatol Alergol 2016;33:397-410

17. Randall KL, Hawkins CA. Antihistamines and allergy. Aust Prescr 2018;41:41-5.

18. Fein MN, Fischer DA, O'Keefe AW, Sussman GL. CSACl position statement: Newer generation $\mathrm{H} 1$-antihistamines are safer than first-generation $\mathrm{H} 1$-antihistamines and should be the first-line antihistamines for the treatment of allergic rhinitis and urticaria. Allergy Asthma Clin Immunol 2019;15:61.

19. Popescu FD. H1 antihistamines and driving. J Med Life 2008;1:262-8.

20. Hox V, Lourijsen E, Jordens A, Aasbjerg K, Agache I, Alobid I, et al. Benefits and harm of systemic steroids for short- and long-term use in rhinitis and rhinosinusitis: an EAACl position paper. Clin Transl Allergy 2020;10:1.

21. Bleecker ER, Menzies-Gow AN, Price DB, Bourdin A, Sweet S, Martin AL, et al. Systematic Literature Review of Systemic Corticosteroid Use for Asthma Management. Am J Respir Crit Care Med 2020;201:276-93.
22. Dvorin EL, Ebell MH. Short-Term Systemic Corticosteroids: Appropriate Use in Primary Care. Am Fam Physician 2020;101:89-94.

23. Streck WF, Lockwood DH. Pituitary adrenal recovery following shortterm suppression with corticosteroids. Am J Med 1979;66:910-4.

24. Aljebab F, Choonara I, Conroy S. Systematic review of the toxicity of shortcourse oral corticosteroids in children. Arch Dis Child 2016;101:365-70.

25. Demoly P, Chabane H, Fontaine JF, de Boissieu D, Ryan D, Angier E, et al. Development of algorithms for the diagnosis and management of acute allergy in primary practice. World Allergy Organ J 2019;12:100022.

26. Pansare M, Seth D, Kamat A, Kamat D. Summer Buzz: All You Need to Know about Insect Sting Allergies. Pediatr Rev 2020;41:348-56.

27. Versporten A, Bolokhovets G, Ghazaryan L, Abilova V, Pyshnik G, Spasojevic T, et al. Antibiotic use in eastern Europe: a cross-national database study in coordination with the WHO Regional Office for Europe. Lancet Infect Dis 2014;14:381-7.

28. Sahin A, Akici A, Aydin V, Melik B, Aksoy M, Alkan A. Variation of antibiotic consumption and its correlated factors in Turkey. Eur J Clin Pharmacol 2017;73:867-73.

29. OECD (2019), Health at a Glance 2019: OECD Indicators, OECD Publishing: Paris. 